\title{
Core Patient-Reported Outcomes (PROs) and PRO Measures (PROMs) for Polypharmacy Medicines Reviews: A Sequential Mixed-Methods Study
}

This article was published in the following Dove Press journal: Patient Preference and Adherence

\author{
Grigorios Kotronoulas (1D) \\ Mark Cooper $\mathbb{D}^{1,2}$ \\ Bridget Johnston ${ }^{1,2}$
}

'School of Medicine, Dentistry \& Nursing, College of Medicine, Veterinary \& Life Sciences, University of Glasgow, Glasgow, UK; ${ }^{2} \mathrm{NHS}$ Greater Glasgow and Clyde, Glasgow, UK
Correspondence: Grigorios Kotronoulas School of Medicine, Dentistry \& Nursing, College of Medicine, Veterinary \& Life Sciences, University of Glasgow, 57-6I Oakfield Avenue, Glasgow G I 2 8LL, United Kingdom

Tel +44 I4I 3306883

Email grigorios.kotronoulas@glasgow.ac.uk
Purpose: Problematic polypharmacy can exaggerate "medicine burden" for the patient. Patient-reported outcomes (PROs) are key indicators of medicine burden, and PRO measures (PROMs) can help patients articulate their perceptions of medicine burden. We aimed to: (a) evaluate what PROMs currently exist that assess medicine burden, and what PROs they target, and (b) understand patients' experiences with using multiple medicines to establish a core set of most meaningful and relevant PROs for assessment in polypharmacy medicines reviews.

Patients and Methods: We conducted a prospective, sequential mixed-methods study in two consecutive work phases. Phase 1 involved a rapid review of PROMs, informed by the published PRISMA and COSMIN initiative guidelines. We integrated all evidence in a thematic narrative synthesis. Phase 2 involved cross-sectional, one-to-one, semi-structured interviews with key stakeholders, including members of the public and healthcare professionals (HCPs). We conducted thematic content analysis to identify and classify emerging PROs.

Results: In Phase 1, 13 studies described the development and/or validation of 12 PROMs. The PROMs targeted 14 content domains of adult patients' experiences with prescribed medicines. PROMs varied widely in terms of length, comprehensiveness and psychometric robustness. In Phase 2, all participants (seven members of the public; eight HCPs) agreed on the clinical relevance of PROMs, providing a rich account of justifications. We identified four core PROs: 'Knowledge, information and communication about own medicines'; "Perceptions, views and attitudes about (own) medicines"; "Impact on daily living: Sideeffects and practicalities", and "Medicine usage: 'as planned', misuse, abuse, no use".

Conclusion: We suggest combining psychometrically robust PROMs or domains across PROMs into a bespoke PROM that addresses comprehensively and succinctly the four core PROs. We recommend a careful implementation process that must involve consultation with all relevant stakeholders, while establishing a clear purpose for collecting a PROM and realistic and ongoing collection at key time-points.

Keywords: medication experiences, preferences, knowledge, perceptions, impact on daily living, monitoring

\section{Plain Language Summary}

Why Was the Study Done? Patients may find taking multiple medicines causes problems with everyday living. These problems are often called "medicine burden". One way to get help for medicine burden is for patients to self-report medicine burden using questionnaires. These are often called patient-reported outcome measures, or PROMs. In this study, we wanted to understand: (a) what PROMs are available and how well they have been developed, and (b) what aspects of medicine burden matter the most. 
What Did the Researchers Do and Find? First, we reviewed the literature and found 12 PROMs. Then, we interviewed patients, family members and clinicians. Interviewees felt that PROMs were well suited to help patients voice their needs. It was agreed that PROMs must monitor four areas of medicine burden, ie, (a) knowledge, information and communication about medicines; (b) perceptions about (one's own) medicines; (c) impact on daily living: sideeffects and practicalities, and (d) actual medicine use.. However, no single PROM fully addressed all four areas.

What Do These Results Mean? To address all of patients' concerns about medicine burden we suggest combining valid PROMs into a bespoke PROM that is easy to understand, concise, and includes only the most essential questions.

\section{Introduction}

Living with multiple chronic illnesses, or multi-morbidity, ${ }^{1}$ often demands the use of multiple medicines on a daily basis. $^{2,3}$ Polypharmacy is a growing phenomenon worldwide, ${ }^{4}$ although often may be clinically warranted. However, coping with polypharmacy in the presence of a long-term illness can be challenging for the patient. ${ }^{5}$ Moreover, "problematic polypharmacy", ie, multiple medicines prescribed inappropriately, can exaggerate the burden for the patient. ${ }^{2}$ This type of burden, known as "medicine burden", has been associated with non-adherence, poor clinical outcomes, and diminished patient well-being and satisfaction with care. ${ }^{6,7}$ However, medicine burden from the patient's viewpoint is still poorly understood, inconsistently measured and often overlooked. ${ }^{8}$

Given the extent of multi-morbidity, ${ }^{9}$ risk for problematic polypharmacy, ${ }^{5}$ and the numbers of people affected, ${ }^{10}$ there is an urgent need to measure and understand medicine burden among people living with multiple chronic illnesses. Patients' experiences of using multiple medicines vary according to number, nature and severity of underlying chronic illnesses; number of medicines and regimen complexity; route and frequency of administration; medicines effectiveness and side-effects; and impact on general wellbeing. $7,11,12$

Patient-reported outcomes (PROs) are key indicators of medicine burden, and PRO measures (or PROMs) can help patients articulate their own outcomes of care, including the benefits and risks of prescribed medicines. ${ }^{8,13}$ PROMs for polypharmacy medicines reviews can allow for quantification of the patient's perceptions of core outcomes and medicines-related experiences. ${ }^{14,15}$ This offers a means to (a) identify patients at greatest risk of harm, (b) screen patients for medicine burden, and (c) evaluate the effectiveness of interventions to tackle problematic polypharmacy and minimise medicine burden.

In Scotland, the Scottish Government's Polypharmacy Guidance for Realistic Prescribing ${ }^{16}$ centralizes the philosophy "that patients are integral to the decisions made about their medicines and [must be] empowered and supported to do so" (p.6) in an attempt to promote appropriate polypharmacy and medicine safety. PROMs can play integral role to this direction. However, before PROMs for polypharmacy medicine reviews are more widely integrated in clinical practice, there is a need to: (a) Understand what PROMs currently exist that assess aspects of medicine burden, and what PROs they target; (b) Evaluate and synthesize the evidence on the psychometric properties of existing PROMs; and (c) Understand patients' experiences with using multiple medicines (to identify emerging PROs), glean views and opinions about the clinical relevance of PROMs, and establish a core set of PROs viewed as most meaningful and relevant for polypharmacy medicines reviews. Our study aimed to address the aforementioned knowledge gaps by answering the following research questions:

RQ1: What PROMs currently exist that assess aspects of medicine burden, and what PROs do they target?

RQ2: What are the reported psychometric properties of these PROMs?

RQ3: What are the experiences of patients with using multiple medicines that can point towards emerging important PROs?

RQ4: What are the views and opinions of stakeholders on the clinical use of PROMs to aid polypharmacy medicines reviews?

RQ5: Which PROs stakeholders view as the most meaningful and relevant to be assessed in polypharmacy medicines reviews?

\section{Materials and Methods}

We conducted a prospective, sequential mixed-methods study that comprised two consecutive work phases, ie, a rapid literature review (Phase 1) and consultation with stakeholders (Phase 2).

\section{Rapid Review (Phase I)}

To answer RQs 1 and 2, we carried out a rapid review of PROMs that target aspects of medicine burden. Our rapid review was informed by the Preferred Reporting Items for Systematic Reviews and Meta-Analyses (PRISMA) 
guidelines ${ }^{17}$ and conducted according to current guidance for rapid reviews. ${ }^{18}$

\section{Search Strategy}

A systematic search strategy was developed in consultation with an academic librarian. Searches were run separately in the following databases: MEDLINE (Ovid), CINAHL (EBSCO), PsycINFO (EBSCO), SocINDEX (EBSCO), Cochrane Library, Web of Science, and Google Scholar. The PROQOLID ${ }^{\circledR}$ database (https://epro vide.mapi-trust.org/) that houses several patient-related measures was searched for additional articles. Reference lists of all included articles were examined. Details of the search terms used for each database can be found in Supplementary file 1 .

Searches were limited to international research published in the English language; eligible studies had to be published between January 1999 and March 2019 to retrieve the most up-to-date evidence. Database searches were further limited to studies published between January 2015 and March 2019 . This was because studies published between January 1999 and January 2015 had already been considered in a previous systematic review ${ }^{8}$ that identified 15 PROMs that evaluated patients' experiences with medicines. Studies and PROMs included in that systematic review were considered for inclusion in our rapid review.

\section{Inclusion/Exclusion Criteria}

Explicit, research-question-driven eligibility criteria were set out, informed by the COnsensus-based Standards for the selection of health Measurement INstruments (COSMIN) initiative guidelines for the selection of PROMs. ${ }^{19}$

\section{Inclusion Criteria}

Eligible studies were those that: (a) Developed and tested a PROM (ie, developed with direct patient input and developed as a self-reported measure) to specifically assess patients' medicine burden and/or patients' experiences with prescribed (multiple) medicines (either as a whole or in a sub-domain /subscale). (b) Involved patients experiencing two or more long-term health conditions (ie, multimorbidity ${ }^{9}$ ) and/or patients receiving multiple medicines (ie, experiencing polypharmacy). (c) Evaluated one or more measurement property and/or the interpretability (ie, distribution of scores, missing items, floor/ceiling effects, change scores) of the PROM under development. We also considered studies that tested/developed revised versions of a pre-existing PROM, and subsequently analyzed original and revised PROM versions, on the proviso that they met all our eligibility criteria.

\section{Exclusion Criteria}

We excluded studies that: (a) Were not concerned with development/testing of a PROM. (b) Developed a PROM that comprised only individual items on medicine-related experiences or burden, ie, items did not form a stand-alone sub-domain/subscale. (c) Used the PROM only to measure its target outcome(s), eg, observational studies or randomized controlled trials. (d) Used a PROM to validate another instrument. (e) Developed/tested a PROM in languages other than English and consequently in cultures other than those where English is the native language. (f) Were (systematic) literature reviews.

\section{Screening and Study Selection}

We transferred retrieved records onto Endnote ${ }^{\circledR}$ reference management software (http://endnote.com/), where they were de-duplicated and subsequently screened on the basis of title and abstract. Retained records were accessed in fulltext, and further screened against our eligibility criteria.

\section{Data Extraction and Synthesis}

We extracted data from the final sample of studies onto a bespoke data extraction form created for this rapid review and inserted into an Excel spreadsheet for ease of use. The data extraction was in line with RQs 1 and 2 to generate information on PROM content, target PROs and psychometric robustness. All evidence was integrated in a thematic narrative synthesis that generated summaries of key PROM elements, content domains and psychometric properties for consideration in Phase 2. A glossary of all psychometric terms used in this study can be found in Supplementary file 2 .

\section{Consultation with Stakeholders (Phase 2)}

To answer RQs 3 to 5, qualitative interviews were conducted with key stakeholders, including members of the public and healthcare professionals.

\section{Study Design}

Descriptive, cross-sectional, qualitative study.

\section{Eligibility and Sampling}

Eligible members of the public were: Adult ( $\geq 18$ years of age) men and women; Diagnosed with $\geq 2$ long-term health conditions or caring for a loved one diagnosed with $\geq 2$ long-term health conditions (ie, multimorbidity ${ }^{9}$ ); Able to speak, write and communicate in English; Not diagnosed with major cognitive or mental disorder that affected communication. 
Eligible healthcare professionals were: General practitioners, practice pharmacists, elderly care consultants, advanced nurse practitioners, and care home staff; Involved in the care of people with multiple chronic illnesses.

Convenience sampling was used as a pragmatic approach to the study's tight timelines. We aimed to conduct up to 20 interviews with members of the public and up to 20 interviews with healthcare professionals to increase the odds for satisfactory diversity in backgrounds and experiences of study participants.

\section{Procedures}

Members of the public were identified and recruited from community groups, charitable organizations or public libraries. We also posted advertisements to Twitter and the dedicated online platform "Call for Participants" (https:// www.callforparticipants.com/). The researcher first checked eligibility with members of the public interested in the study, then offered specific information using a Participant Information Sheet and Privacy Notice. The researcher offered to clarify any points and answer possible questions.

Healthcare professionals were recruited via diverse routes, including Twitter and non-NHS professional meetings and conferences. We also used a snowballing technique whereby healthcare professional participants invited colleagues via their networks to consider participation. We sent healthcare professionals interested in taking part a Participant Information Sheet and Privacy Notice to their email address. The researcher again offered to clarify any points and answer possible questions.

Potential participants were free to refuse without a requirement to justify their decision and without any penalty. If an eligible member of the public agreed or healthcare professional wished to take part in the study, we provided him/her with a consent form to sign and return, and then agreed a suitable date/time and mode for the interview. Research participants were free to withdraw at any point if they so wished without a requirement to justify their decision.

\section{Data Collection}

Sequential, one-to-one, semi-structured interviews were conducted by the lead author or a research assistant. We conducted the interviews in a sequential fashion, depending on research participants' earliest availability. We offered diverse interview modes, ie, face-to-face interviews or telephone/ Skype interviews, in order to facilitate easier recruitment/ data collection. The interview took place at a time (and place where applicable) most convenient to the research participant. We anticipated that interviews would be 30-60 mins long, with a mean duration of 45 mins.

In preparation for the interviews, we used findings from the rapid review to construct an interview guide to allow the systematic exploration of stakeholders' opinions/views. Before any given interview, we sent each research participant a summary sheet to ensure basic understanding of the concepts of polypharmacy, medicine burden and PROMs, and key findings from the rapid evidence review. At the end of the interview, we debriefed the research participant and thanked him/her for their time and contribution.

\section{Data Analysis}

Thematic content analysis was undertaken. ${ }^{20}$ Thematic content analysis is a useful approach for answering questions about the salient issues for a particular group of respondents or for identifying typical responses. ${ }^{20}$ The analysis of transcripts began with familiarization. The lead author identified themed categories based on the research objectives, which were subsequently verified by the co-authors. Using the three pre-set conceptual categories (experiences with using medicines, views/opinions about PROMs, and most meaningful/important PROs) to serve as an initial set of themes, we established a framework in which these three overarching themes served as larger elements into which emerging concepts were categorized as sub-themes.

\section{Results}

\section{Rapid Review (Phase I)}

Search Results

After completing the initial identification and screening process, we retained and retrieved in full-text 71 potentially eligible articles. Of these, we excluded 58 for various reasons (see Figure 1). Thirteen studies met our eligibility criteria and comprised our final sample. These studies described the development and/or validation of 12 PROMs. Access links to these PROMs can be found in Supplementary file 3.

\section{Overview of Identified PROMs}

Three measures were different versions of the Living with Medicines Questionnaire (LMQ; ${ }^{21,22}$ LMQ-2; ${ }^{23}$ $\mathrm{LMQ}^{24}{ }^{24}$. Two were different versions of the Treatment Satisfaction Questionnaire for Medication (TSQM v1; ${ }^{25}$ TSQM v2 $\left.{ }^{26}\right)$. Another two were different versions of the Patients' Attitudes Towards Deprescribing (PATD ${ }^{27}$ $\left.\operatorname{rPATD}^{28}\right)$. 



Figure I PRISMA flow diagram of article screening and selection procedures (adapted from Moher et al, $2009^{17}$ ).

The remaining five were single-version measures, including the following: Beliefs about Medicines Questionnaire $\left(\mathrm{BMQ}^{29}\right)$; Medication Use Questionnaire $\quad\left(\right.$ MedUseQ $\left.{ }^{30}\right) ; \quad$ Medication-Related
Burden Quality of Life (MRB-QoL $\left.{ }^{31}\right)$; Patient Experience with Treatment and Self-Management $\left(\mathrm{PETS}^{32}\right)$; and Patient Perceptions of Deprescribing Questionnaire $\left(\mathrm{PPDQ}^{33}\right)$. 


\section{Origin, Population and Setting}

Five PROMs were developed in the USA (MedUseQ; ${ }^{30} \mathrm{MS}-$ PETS; ${ }^{32}$ PPDQ; ${ }^{33}$ TSQM v1; ${ }^{25}$ TSQM v2 ${ }^{26}$ ), four were developed in the UK (BMQ; ${ }^{29}$ LMQ; ${ }^{21,22}$ LMQ-2; ${ }^{23}$ $\mathrm{LMQ}^{24}$ ), and three in Australia (PATD; ${ }^{27}$ rPATD; ${ }^{28} \mathrm{MRB}-$ QoL $\left.{ }^{31}\right)$.

The PROMs were developed primarily with chronically ill adults ( $>18$ years), using one or more long-term prescribed medicines. Study participants were recruited from hospitals, community pharmacies, senior housing facilities or community-based organizations for older adults, health websites/social media, or the general public.

Statistics about multimorbidity, ie, medians ( $\geq 2$ concurrent chronic conditions per person), or references to "patients with multiple chronic conditions" were explicitly given for the MRB-QoL, ${ }^{31}$ PATD, ${ }^{27}$ and MS-PETS. ${ }^{32}$

Statistics about polypharmacy, ie, rates (40-93.5\%) or medians ( $\geq 5$ concurrent prescribed medicines per participant), were explicitly reported for the LMQ-2, ${ }^{23} \mathrm{LMQ} 3,{ }^{24}$ MedUseQ, ${ }^{30}$ MRB-QoL, ${ }^{31}$ MS-PETS, ${ }^{32}$ PPDQ, ${ }^{33}$ and rPATD. $^{28}$ In all other studies, numbers of comorbid conditions per participant and/or of concurrent medicines per participant were either not reported or fell below the multimorbidity/polypharmacy thresholds.

\section{User Involvement}

Direct user (patients and clinicians) involvement at both the "item generation" and "item clarification" stages took place for the LMQ, ${ }^{21,22}$ LMQ-2, ${ }^{23}$ MedUseQ, ${ }^{30}$ MSPETS, ${ }^{32}$ PPDQ ${ }^{33}$ rPATD, ${ }^{28}$ and TSQM v1. ${ }^{25}$

For the $\mathrm{BMQ},{ }^{29}$ direct user involvement took place at the "item generation" stage only. For the LMQ3, ${ }^{24} \mathrm{MRB}-$ QoL, ${ }^{31}$ PATD, ${ }^{27}$ and TSQM v2, ${ }^{26}$ direct user involvement took place at the "item clarification" stage only.

User involvement was in the form of focus group or one-to-one interviews, concept mapping exercises, expert panels, and cognitive interviews.

\section{Focus Area, Domains and Dimensionality}

The PROMs tapped into five broad areas of focus, namely medicine burden (LMQ; ${ }^{21,22}$ LMQ-2; ${ }^{23}$ LMQ3; ${ }^{24}$ MRBQoL; ${ }^{31}$ PETS $^{32}$ ); attitudes towards deprescribing (PATD; ${ }^{27}$ rPATD ${ }^{28}$ PPDQ $^{33}$ ); satisfaction with medicines (TSQM $\left.\mathrm{v} 1 ;^{25} \mathrm{TSQM} \quad \mathrm{v} 2^{26}\right) ;$ medicine use problems $\left(\right.$ MedUseQ $\left.{ }^{30}\right)$; and beliefs towards medicines $\left(\mathrm{BMQ}^{29}\right)$.

Within their areas of focus, nine PROMs targeted diverse domains (LMQ; ${ }^{21,22}$ LMQ-2; ${ }^{23}$ LMQ3; ${ }^{24}$ MRBQoL $;^{31}$ PETS; ${ }^{32}$ rPATD ${ }^{28}$ PPDQ; ${ }^{33}$ TSQM v1; ${ }^{.25}$ TSQM $\mathrm{v} 2^{26}$ ). Three PROMs covered only one domain (PATD; ${ }^{27}$ MedUseQ; $\left.{ }^{30} \mathrm{BMQ}^{29}\right)$; however, the $\mathrm{BMQ}^{29}$ was divided into subscales.

In terms of dimensionality, nine PROMs featured between four and ten subscales LMQ; ${ }^{21,22}$ LMQ-2; ${ }^{23}$ LMQ3; ${ }^{24}$ MRB-QoL; ${ }^{31}$ rPATD; ${ }^{28}$ PPDQ; ${ }^{33}$ TSQM v1; ${ }^{25}$ TSQM v2; ${ }^{26} \mathrm{BMQ}^{29}$ ). The $\mathrm{PETS}^{32}$ is a multi-dimensional measure of treatment burden comprising nine subscales, but only the Medicines subscale was relevant to the remit of this review, and thus specifically targeted (thereafter, referred to as MS-PETS ${ }^{32}$ ). The MedUseQ ${ }^{30}$ is a psychometrically confirmed unidimensional measure, whereas the PATD ${ }^{27}$ has no confirmed dimensionality.

\section{Domain Content: Target PROs}

Collectively, the PROMs covered 14 unique domains (Table 1). Owing to the developmental work of the PROMs involving the direct patient and public involvement, these domains most probably reflect most issues that affect people using regular medicines.

The top-3 domains based on the frequency of coverage across the PROMs were: Impact on daily living due to medicine use (including medicine burden); Convenience, practicalities and/or managing medicines (including support); and Views, attitudes, beliefs and/or perceptions about medicines (Table 1). The LMQ3, ${ }^{24}$ MedUseQ, ${ }^{30}$ and $\operatorname{rPATD}^{28}$ were the most comprehensive measures, each covering 10 domains.

\section{Scale Item Range}

All PROMs were multi-item, ranging from seven to 43 items per measure. The only measure with fewer than 10 items was the MS-PETS. ${ }^{32}$ Five measures included 11-20 items (BMQ; ${ }^{29}$ PATD; ${ }^{27}$ PPDQ; ${ }^{33}$ TSQM v1; ${ }^{25}$ TSQM v2 ${ }^{26}$ ). Two measures included 21-30 items (MedUseQ; ${ }^{30}$ rPATD $\left.^{28}\right)$. Four measures comprised $30+\left(\mathrm{MRB}-\mathrm{QoL}^{31}\right)$ or $40+$ items (LMQ; ${ }^{21,22}$ LMQ-2; ${ }^{23}$ LMQ3 $^{24}$ ).

\section{Response Format and Scoring}

Ten measures used a 5-point or 7-point Likert scale for respondents to indicate agreement/disagreement with statements $_{\text {(BMQ }}{ }^{29}$ LMQ; ${ }^{21,22}$ LMQ- $2 ;^{23}$ rPATD ${ }^{28}$ PPDQ $^{33}$ MRB-QoL; ${ }^{31}$ TSQM v1; ${ }^{25}$ TSQM v2; ${ }^{26}$ LMQ3; ${ }^{24}$ PATD $^{27}$ ). Numerical scales (MedUseQ; ${ }^{30}$ MSPETS; ${ }^{32}$ TSQM v1; ${ }^{25}$ TSQM v2 ${ }^{26}$ ), visual analogue scales $\left(\mathrm{LMQ}^{24}\right)$, or multiple-choice questions $\left(\mathrm{PATD}^{27}\right)$ were used less frequently. 


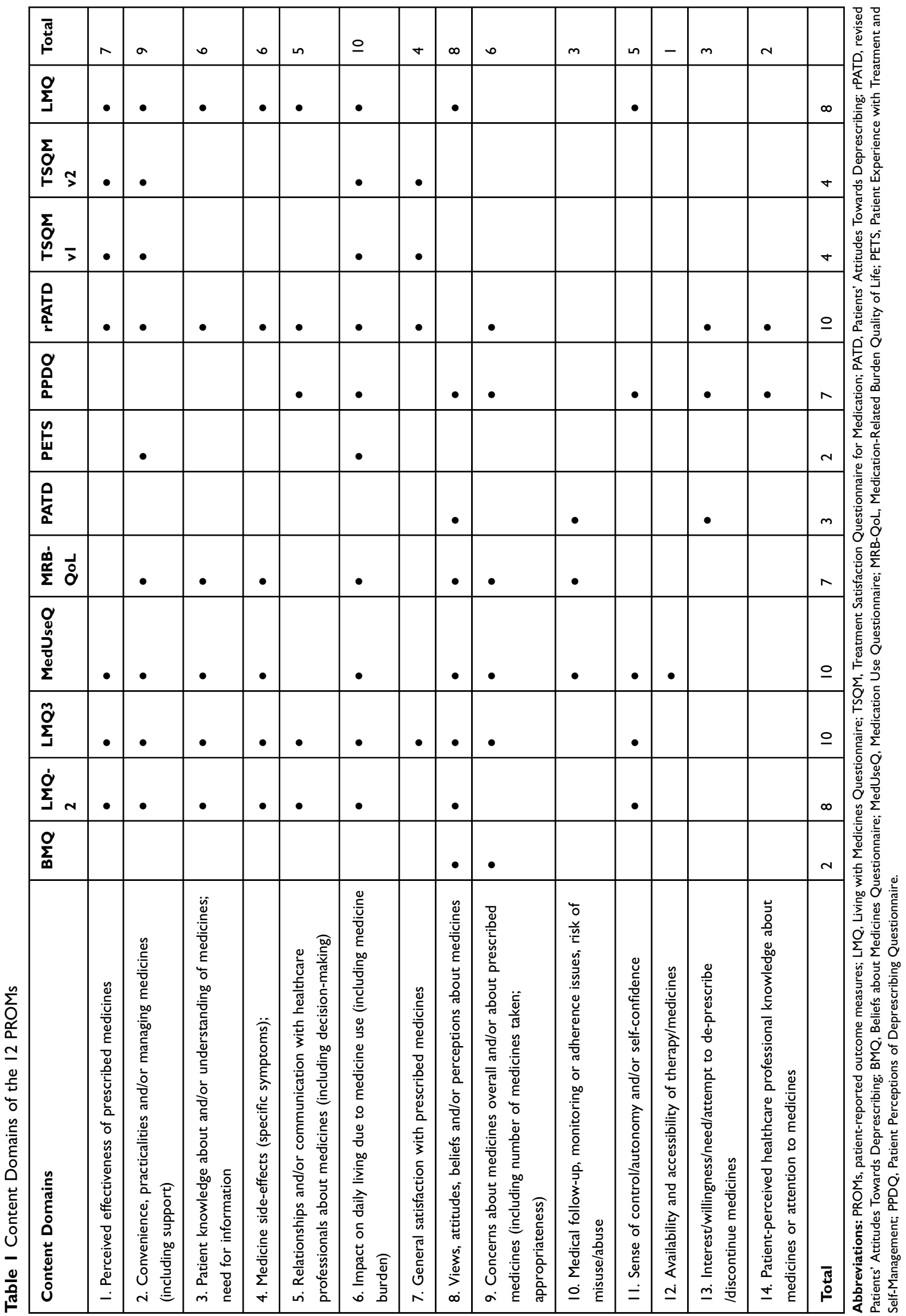


In terms of scoring, eight PROMs employed one scoring system (BMQ; ${ }^{29}$ LMQ; ${ }^{21,22}$ LMQ-2; ${ }^{23}$ MedUseQ; ${ }^{30}$ rPATD $;^{28}$ MS-PETS; ${ }^{32}$ PPDQ; ${ }^{33}$ MRB-QoL ${ }^{31}$ ). Four measures employed two scoring systems (TSQM v1; $;{ }^{25} \mathrm{TSQM}$ $\mathrm{v} 2 ;{ }^{26} \mathrm{LMQ} 3 ;{ }^{24} \mathrm{PATD}^{27}$ ). Two measures included an openended field (LMQ; ${ }^{21,22} \mathrm{MRB}^{-\mathrm{QoL}^{31}}$ ). For three PROMs (BMQ; ${ }^{29}$ LMQ-2; ${ }^{23}$ LMQ $^{24}$ ) both subscale and total scores can be calculated. Seven PROMs yield subscale scores only (MRB-QoL; ${ }^{31}$ MS-PETS; ${ }^{32}$ rPATD; ${ }^{28}$ PPDQ ${ }^{33}$ LMQ $;{ }^{21,22}$ TSQM v1; ${ }^{25}$ TSQM v2 ${ }^{26}$ ). For the MedUse ${ }^{30}$ a single total score can be calculated, whereas for the PATD ${ }^{27}$ no total score can be calculated.

\section{Recall Period}

Seven PROMs involve generic items with no specific timeframe for recall (BMQ; ${ }^{29}$ LMQ; ${ }^{21,22}$ LMQ- $2 ;^{23}$ LMQ $3{ }^{24}$ PATD $;{ }^{27}$ PATD ${ }^{28}$ PPDQ $\left.^{33}\right)$. Recall period for five measures ranged from "past 2 weeks" (MRB-QoL ${ }^{31}$ ) to "past 2-3 weeks" (TSQM v1; ${ }^{25}$ TSQM v2 ${ }^{26}$ ) to "past 4 weeks" (MS-PETS ${ }^{32}$ ) to "past 3 months" (MedUseQ ${ }^{30}$ ).

\section{Psychometric Robustness}

\section{Reliability: Internal Consistency and Stability}

Internal consistency was reported for all PROMs except for the LMQ. ${ }^{21,22}$ Measurement of this property relied on the calculation of two sets of metrics: Cronbach's alpha and/or inter-item, item-to-total or inter-scale correlations. Cronbach's alphas varied widely across subscales of the same measure or across different measures. Consistently, the MedUseQ, ${ }^{30}$ MRB-QoL, ${ }^{31}$ MS-PETS, ${ }^{32}$ TSQM v1, ${ }^{25}$ and TSQM v $2^{26}$ had alphas at or above the 0.80 threshold for all of their subscales. For the LMQ $3^{24}$ and rPATD, ${ }^{28}$ Cronbach's alphas were acceptable (ie. $\geq 0.80$ ) for most of their individual subscales.

Stability (or test-retest) was tested only for the BMQ, ${ }^{29}$ LMQ3, ${ }^{24}$ PATD,${ }^{27}$ and rPATD. ${ }^{28}$ Investigation included calculation of parametric/non-parametric correlation coefficients or intraclass correlation coefficients (ICC), percentage agreement, or test-retest mean differences. Retest intervals ranged from 3 days $\left(\mathrm{PATD}^{27}\right)$ to one $\left(\mathrm{rPATD}^{28}\right)$ or 2 weeks $\left(\mathrm{BMQ},{ }^{29} \mathrm{LMQ}^{24}\right)$. Stability metrics were acceptable (ICC $\geq 0.75 ; \geq 80 \%$ agreement; no statistically significant mean differences at retest) for all four PROMs; however, analyses were based on small samples $(n=\sim 30)$.

\section{Content Validity}

Content validity of the PROMs was established by means of literature reviews and/or stakeholder input. Development of the LMQ, ${ }^{21,22}$ LMQ-2 ${ }^{23}$ and TSQM v2 ${ }^{26}$ relied solely on patient and public involvement. For the development of the $\mathrm{BMQ}^{29}$ and TSQM v1, ${ }^{25}$ literature reviews were complemented by patient input.

Development of the LMQ3 $3{ }^{24}$ MedUseQ, ${ }^{30}$ MRB-QoL, ${ }^{31}$ PETS, ${ }^{32}$ PATD,${ }^{27}$ PATD $^{28}$ and PPDQ ${ }^{33}$ combined extensive literature reviews with patient and clinician input from expert panels and focus groups. For item clarification, cognitive interviews (LMQ, ${ }^{21,22} \mathrm{LMQ}^{24}{ }^{24} \mathrm{MedUseQ},{ }^{30} \mathrm{PPDQ}^{33}$ ) or pilot trials with patients (PATD, ${ }^{27}$ rPATD, ${ }^{28}$ TSQM v1 ${ }^{25}$ ) were also conducted.

\section{Construct Validity}

Construct validity of the PROMs was investigated as part of scale analysis and/or known-groups validity.

Development of most PROMs employed exploratory factor analysis techniques for scale analysis (BMQ ${ }^{29}$ LMQ, ${ }^{21,22}$ LMQ-2, ${ }^{23}$ LMQ3, ${ }^{24}$ MedUseQ, ${ }^{30}$ MRBQoL, ${ }^{31}$ PETS, ${ }^{32}$ PPDQ,${ }^{33}$ rPATD, ${ }^{28}$ TSQM v1, ${ }^{25}$ TSQM $\mathrm{v}^{26}$ ). Confirmatory methods to ascertain underlying content domains were used less frequently (BMQ, ${ }^{29}$ LMQ, ${ }^{21,22}$ LMQ3, ${ }^{24}$ PETS, ${ }^{32}$ PPDQ, ${ }^{33}$ TSQM v2 ${ }^{26}$ ). No scale analysis was conducted for the PATD. ${ }^{27}$

Known-groups validity was confirmed via significant between-group differences for six PROMs, namely: $\mathrm{BMQ}^{29}$ (allopathic v. complementary care group); LMQ$2^{23}$ (1-4 v. 5-8 v. $\geq 9$ concurrent medicines); LMQ3 ${ }^{24}$ (higher scores for patient groups who took $5+$ medicines, took medicines 3 times daily, required assistance with taking medicines, paid for prescriptions); MRB-QoL ${ }^{31}$ (higher scores for patient groups with polypharmacy, multimorbidity, and drug burden index $>0)$; $\operatorname{PATD}^{27}(0-5$ v. 6-9 v. 10+ medicines); and PETS ${ }^{32}$ (higher scores for patient groups with lower health literacy, less adherence to medicines, and more financial difficulties). There was conflicting evidence reported for the $\operatorname{rPATD}^{28}$ and TSQM $\mathrm{v} 1 .^{25}$

\section{Criterion Validity}

For most PROMs, criterion validity was established by investigating either one or both of its two aspects, ie, concurrent validity (convergent and/or discriminant validity) and predictive validity.

Convergent validity was established for the $\mathrm{BMQ},{ }^{29}$ LMQ3 ${ }^{24}$ MRB-QoL,${ }^{31}$ PATD,${ }^{27}$ rPATD ${ }^{28}$ MS-PETS, ${ }^{32}$ TSQM v1, ${ }^{25}$ and TSQM v2 ${ }^{26}$ via statistically significant relationships of these PROMs with other conceptually similar 
measures. Discriminant validity was established only for the MRB-QoL. ${ }^{31}$

Concurrent validity was more fully examined for the MRB-QoL. ${ }^{31}$ For four PROMs, no evidence on concurrent validity is available (LMQ, ${ }^{21,22}$ LMQ- $2,{ }^{23}$ MedUseQ, ${ }^{30}$ $\mathrm{PPDQ}^{33}$ ).

Predictive validity was tested only for three PROMs, ie, $\mathrm{BMQ}^{29}$ (beliefs about medicines predicted greater adherence to medicine use), TSQM v1 ${ }^{25}$ (satisfaction with medicines predicted likelihood to discontinue medicines), and TSQM $\mathrm{v} 2^{26}$ (satisfaction with medicines predicted medicine persistence/completeness).

\section{Floor/Ceiling Effects}

Floor and/or ceiling effects were reported for eight PROMs. Floor effects were reported for the LMQ3 ${ }^{24}$ (five items affected; floor effects at max. 59.1\%) and MedUseQ ${ }^{30}$ (across all items; floor effects at 9.8\%). Ceiling effects were reported for the LMQ- $2^{23}$ (one item affected; ceiling effects at $68.5 \%$ ), TSQM v1 ${ }^{25}$ (across subscales; ceiling effects at 8.9-41.1\%, global satisfaction; ceiling effects at $12.9 \%$ ), and TSQM v2 ${ }^{26}$ (across subscales; ceiling effects at $8.2-76.7 \%$, overall satisfaction; ceiling effects at $14.3 \%$ ).

A mix of floor and ceiling effects were reported for the: MRB-QoL ${ }^{31}$ (Social burden subscale; floor effects at 0.6$19 \%$, Therapeutic relationship subscale; ceiling effects at 1.7-24.8\%); MS-PETS ${ }^{32}$ (across all items; floor effects at $38-59 \%$, ceiling effects at $0 \%$; Stand-alone medicines items: Reliance, floor effects at $46-65 \%$, ceiling effects at $1-14 \%$; Side-effects, floor effects at $53-71 \%$, ceiling effects $1-3 \%$ ); and PPDQ $^{33}$ (unimportance of medicines subscale, floor effects at $0.76-6.8 \%$, Provider knowledge subscale, ceiling effects at $0-12.5 \%)$.

\section{Consultation with Stakeholders (Phase 2)}

Between May and June 2019, 12 members of the public and 11 healthcare professionals expressed interest in the study. Seven members of the public $(\mathrm{RR}=58.3 \%)$ and eight healthcare professionals $(\mathrm{R} R=72.7 \%)$ provided written informed consent and completed an interview. Interviews with members of the public lasted for a mean of 42.4 mins (22-58 mins); the mean duration of interviews with healthcare professionals was 30 mins (19-48 mins). Background characteristics of all participants are shown in Tables 2-4.

We extracted three overarching themes, underpinned by within-theme sub-themes: Experiences with using multiple medicines - Emerging PROs (Theme 1); Relevance, potential uses and handlers of PROMs (Theme 2); Most meaningful PROs for medicines reviews (Theme 3). Representative quotes can be found in $\underline{\text { Supplementary file } 4 .}$

Table 2 Background Characteristics of the Study Participants

\begin{tabular}{|c|c|c|c|}
\hline $\begin{array}{l}\text { Participant } \\
\text { Group }\end{array}$ & Variable & Attribute & Value \\
\hline \multirow{16}{*}{$\begin{array}{l}\text { Members of } \\
\text { the public }\end{array}$} & Gender & Male:female & $3: 4$ \\
\hline & Role & Patient:carer & $6: 1$ \\
\hline & \multirow[t]{3}{*}{ Age (years) } & Mean \pm SD & $\begin{array}{l}39.5 \\
\pm 20.3\end{array}$ \\
\hline & & Median & 32 \\
\hline & & Min-Max & $22-78$ \\
\hline & \multirow[t]{3}{*}{ Family status } & Married/partnered & 2 \\
\hline & & Single & 3 \\
\hline & & Widowed & 2 \\
\hline & \multirow{4}{*}{$\begin{array}{l}\text { Employment } \\
\text { status }\end{array}$} & Employed (full-time) & 3 \\
\hline & & Employed (part-time) & 2 \\
\hline & & Retired & 2 \\
\hline & & Student & I \\
\hline & \multirow{2}{*}{$\begin{array}{l}\text { Chronic } \\
\text { conditions }\end{array}$} & Median & 4 \\
\hline & & Min-Max & $2-6$ \\
\hline & \multirow{2}{*}{$\begin{array}{l}\text { Prescribed } \\
\text { medicines }\end{array}$} & Median & 7 \\
\hline & & Min-Max & $3-16$ \\
\hline \multirow{10}{*}{$\begin{array}{l}\text { Healthcare } \\
\text { professionals }\end{array}$} & Gender & Male:female & $6: 2$ \\
\hline & \multirow[t]{5}{*}{ Clinical role } & General practitioner & 3 \\
\hline & & Consultant & I \\
\hline & & Pharmacist & 2 \\
\hline & & Pharmacy technician & I \\
\hline & & $\begin{array}{l}\text { Advanced nurse } \\
\text { practitioner }\end{array}$ & I \\
\hline & \multirow{3}{*}{$\begin{array}{l}\text { Clinical } \\
\text { experience } \\
\text { (years) }\end{array}$} & Mean $\pm S D$ & $\begin{array}{l}22.6 \\
\pm 8.1\end{array}$ \\
\hline & & Median & 25 \\
\hline & & Min-Max & $10-30$ \\
\hline & Area of practice & Urban:rural & $5: 3$ \\
\hline
\end{tabular}

Abbreviation: SD, standard deviation. 
Table 3 Breakdown of Characteristics of Members of the Public

\begin{tabular}{|l|l|l|l|l|l|l|l|}
\hline Study ID & Gender & $\begin{array}{l}\text { Age } \\
\text { (Years) }\end{array}$ & $\begin{array}{l}\text { Family } \\
\text { Status }\end{array}$ & Work Status & $\begin{array}{l}\text { Conditions } \\
\text { (n) }\end{array}$ & $\begin{array}{l}\text { Prescribed Meds } \\
\text { (n) }\end{array}$ & $\begin{array}{l}\text { Interview Duration } \\
\text { (Minutes) }\end{array}$ \\
\hline MP-0I & Female & 24 & Single & Student & 5 & 5 & 27 \\
MP-03 & Male & 22 & Single & Employed $(\mathrm{p} / \mathrm{t})$ & 2 & 3 & 22 \\
MP-04 & Female & 29 & Partnered & Employed $(\mathrm{f} / \mathrm{t})$ & 3 & 8 & 51 \\
MP-06 & Female & 56 & Widowed & Retired & 5 & 7 & 50 \\
MP-08 & Male & 32 & Single & Employed $(\mathrm{f} / \mathrm{t})$ & 3 & 5 & 36 \\
MP-10 & Male & 36 & Married & Employed $(\mathrm{p} / \mathrm{t})$ & 6 & 16 & 58 \\
MP-12 & Female & 78 & Widowed & Retired & 4 & 10 & 53 \\
\hline
\end{tabular}

Abbreviations: MP, member of the public; $p / t$, part-time; f/t, full-time.

Table 4 Breakdown of Characteristics of Healthcare Professionals

\begin{tabular}{|l|l|l|l|l|l|}
\hline Study ID & Gender & Clinical Role & Clinical Experience (Years) & Area of Practice & Interview Duration (Minutes) \\
\hline HCP-0I & Male & GP & 20 & Semi-rural & 19 \\
HCP-02 & Male & GP & 30 & Urban & 24 \\
HCP-03 & Male & GP & 12 & Semi-rural & 48 \\
HCP-05 & Male & Consultant geriatrician & 28 & Urban & 28 \\
HCP-06 & Female & Pharmacist & 30 & Urban & 35 \\
HCP-07 & Male & Pharmacy technician & 10 & Rural & 20 \\
HCP-08 & Female & Pharmacist & 29 & Urban & 47 \\
HCP-II & Male & ANP & 22 & Urban & 19 \\
\hline
\end{tabular}

Abbreviations: HCP, healthcare professional; GP, general practitioner; ANP, advanced nurse practitioner.

Theme I: Experiences with Using Multiple Medicines - Emerging PROs

Within Theme 1, we identified seven sub-themes that comprehensively described the self-reported or healthcare professional-reported experiences of members of the public with multiple medicines.

\section{Social Pressure, Embarrassment and Stigma}

Members of the public talked about a general sense of unease, embarrassment and perceived stigma when managing, taking or talking about medicines in front of or with others. In certain cases, such feelings were fueled by societal, cultural or family attitudes towards medicine use and links with overt or covert pitifulness due to ill health, or assumptions about mental illness or addiction.

\section{Navigating the Prescribing System}

Collectively, participants shared their concerns or frustrations regarding dealing with a system that seems fragmented and not synchronized. Members of the public talked about being under multiple different teams that do not always seem to communicate well. This kind of separation was perceived as a "big divide" that only creates confusion about what services deal with what, particularly in relation to transitions from primary to secondary care and vice versa.

As a result, patients often feel unable to follow-up changes in their treatment protocol made from different prescribers, and particularly worry about shortage or unavailability of medicines or dosages in this complex environment. Participants also talked about "legacy regimens" that might remain untouched for a long time before they are reviewed, possible reluctance to change ineffective regimens for fear of stepping onto other prescribers' toes, and prescriptions that might not be reviewed due to sheer lack of resources.

Multiple Medicines, Side Effects and Interactions: Number V. Impact

A distinction between number of medicines and impact of side effects was evident in the interviews. Participants felt that side effects and/or interactions were not necessarily linearly related to increasing numbers of medicines. Side effects and/or interactions (being one of the many layers of medicine burden) were seen as unique, stressful events that might be attributed to one or two medicines only, although which ones exactly among multiple medicines could be hard to decipher. 
At the same time, a sense of concern and anticipatory anxiety about possible side effects and/or interactions was described, particularly in relation to newly prescribed medicines. Despite this evident impact, an interesting viewpoint was shared that some people might be keen to withstand any side effects in the hope of medicine effectiveness.

\section{Managing (with) Medicines on a Day-to-Day Basis}

Participants dwelled on a range of practicalities related to managing (with) medicines in everyday life. Taking multiple medicines in one go can "be a pain" and finding the "right" form (eg, tablet or capsule) can take a bit of experimenting. Moreover, dealing with different packs and bottles and medicines of the same color and shape can "mix you up", following instructions for different medicines can be confusing, and carrying medicines about can be impractical. Participants also talked about cases where family members seemed to struggle with the responsibility of planning and administering medicines without help. Discussion also revolved around restrictions and a lack of spontaneity in life that medicines seem to pose.

The general impression was that adjusting one's lifestyle and socializing can often be a struggle, while arranging holidays and travelling might be compromised unless well-planned in advance. In terms of planning, one participant discussed the likely implications of residential remoteness to getting access to medicines.

Members of the public also talked about how reducing the amount of medicines can make a "massive difference", and how this might increase their sense of being in control and their feelings of empowerment. Similarly, little practical aids, such as the use of dosette boxes, together with self-confidence and help from the family could increase concordance to the treatment plan and reduced the risk for overuse or abuse.

\section{Getting to Know Your Medicines}

Members of the public agreed that understanding one's own medicines means the healthcare professional takes the time to consult and provide clear explanation. The general feeling was that much more must be discussed about medicines, but that is not always the case, and this might be seen as people being left to deal with medicines on their own. One doing their own research (mainly, online) was another way of getting at least some information. In any case, members of the public collectively voiced a need for better understanding about what medicines do, about the need for those prescribed medicines, about possible side effects and interactions, and about timing and mode of administration.

Healthcare professionals also agreed on the requirement to adequately educate patients and families to develop a sense of ownership over their medicines. Based on their clinical experiences, common issues were a lack of understanding about: what the prescribed medicines are for; the duration, importance, and risks of not following the treatment plan; the intended benefit and effectiveness of a given medicine for the person; the need for regular medicines reviews; and whether and why multiple medicines are appropriate or not.

Additional important views revolved around: patients having set beliefs and opinions about how medicines work and how they should be used; dealing with people who do not want to know or be involved in decision-making; and dosette boxes making people less aware of their own medicines despite their practical aspects. The potential link between understanding the effectiveness of a given medicine and giving up fixed and unhelpful beliefs about medicines was also discussed; this can subsequently increase the odds for the patient to follow the treatment plan and their satisfaction with medicines.

\section{Getting off Medicines}

In the interviews, a distinction was made apparent between patient vs. healthcare professional driven discontinuation. Members of the public indicated that their wishes to reduce or stop a medicine should be followed by an honest discussion with the healthcare professional, and be appropriately followed up and monitored.

For healthcare professionals, experienced side effects and people's initial acceptance of the treatment plan might drive people's desire to reduce medicines. Although not normally directly raised by patients, if the healthcare professional broaches the topic, patients seem open to discuss. However, it was suggested that, where discontinuation is healthcare professional driven, providers must first build trust and approach the topic sensitively in order to curb possible resistance from patients and families concerned about stopping or changing medicines.

Involvement and Engagement in Decisions About Medicines Building rapport and relationship, while working in partnership, were key aspects of involving people in decisions about medicines. Two members of the public were critical towards 
how inclusive some healthcare professionals seem to be despite a clear appetite for involvement from patients or family members.

In their interviews, healthcare professionals agreed that the top limiting factor is not the patient's appetite to be involved, but probably the lack of time in the system to allow a thorough discussion, or even a screening process about who wants to be involved and who does not. In any case, using language acceptable to people (eg, agreement to plan of treatment instead of adherence or compliance) and letting people consider how confident or now they feel to implement an agreed treatment plan were suggested as factors promoting engagement.

\section{Theme 2: Relevance, Usability, Usage and Potential Handlers of PROMs}

Four sub-themes were included in Theme 2, describing various aspects of implementing PROMs in clinical practice to aid polypharmacy medicines reviews.

\section{Perceived Relevance in Clinical Practice}

All participants agreed on the clinical relevance of PROMs to aid medicines reviews, discussing a wide range of potential benefits. Members of the public felt that PROMs could: standardize the consultation; help healthcare professionals gain a deeper understanding of the patient's and family's perspective; act as reminders for people to raise issues during the consultation; enhance relationships and communication between patients and healthcare professionals; and facilitate transfer of information to allow better communication across healthcare professionals. Moreover, PROMs were facilitators of future planning; enablers of improved knowledge on the purpose and actions of medicines; vehicles to legitimize people's concerns; and promoters of honesty in people's reports about side effects and attempts to discontinue medicines.

Healthcare professionals considered PROMs as a "good starting point" to help them understand people's perceptions, preferences and concerns by asking key questions; help them communicate the intended benefit of prescribed medicines, thus increasing people's understanding of their medicines; and help people to actively engage with and take ownership over their medicines, thus increasing their involvement in decision making.

\section{Usability Aspects}

Brevity and clarity were the most important usability aspects of PROMs raised in the interviews. Participants suggested that implementable PROMs for medicines reviews must be quick to fill out (in about 5 mins); include only those essential/meaningful questions; allow people to expand on their responses; provide the option of paper or electronic version; be user-friendly, easily understood and not too wordy; and take health literacy into account.

\section{Delivery, Timing and Frequency}

Regarding usage of PROMs, participants' suggestions ranged widely from weekly to at least once a year. Five members of the public favored a monthly administration. For others, delivery of PROMs could be tied to changes to the treatment plan, introduction of a new medicine, or follow up after problems experienced with a given medicine. One participant suggested that copies of PROMs be available at the surgery or pharmacy or online to allow prompt reporting. What was felt as particularly important was that PRO information be made available to all healthcare professionals involved in one's treatment plan.

Healthcare professionals endorsed an on-going process of collecting PROMs. They also favored a delivery mode were PROMs are filled out beforehand prior to the consultation to allow people to do some homework and healthcare professionals to prepare. A sensitive approach and one that ensures anonymity was also suggested to foster honesty in people's responses. To enable uptake of PROMs a buy-in process for patients and healthcare professionals would need to take place, whereby patients can easily understand the purpose of the PROMs, while healthcare professionals are supported to counterbalance the volume of information gleaned with the time available to review.

\section{Potential Handlers of PRO Information}

Among participants, pharmacists (particularly, community pharmacists) were considered as most appropriate to distribute, collect and review PROMs for medicines reviews. A role for nurses and GPs was also suggested. However, the general consensus was that, regardless of who is involved, the process must be properly and carefully planned.

\section{Theme 3: Most Meaningful PROs for Medicines Reviews}

Within Theme 3, we identified four sub-themes that described the most meaningful PROs to be assessed in medicines reviews (Figure 2).

Knowledge, Information and Communication About Own Medicines 


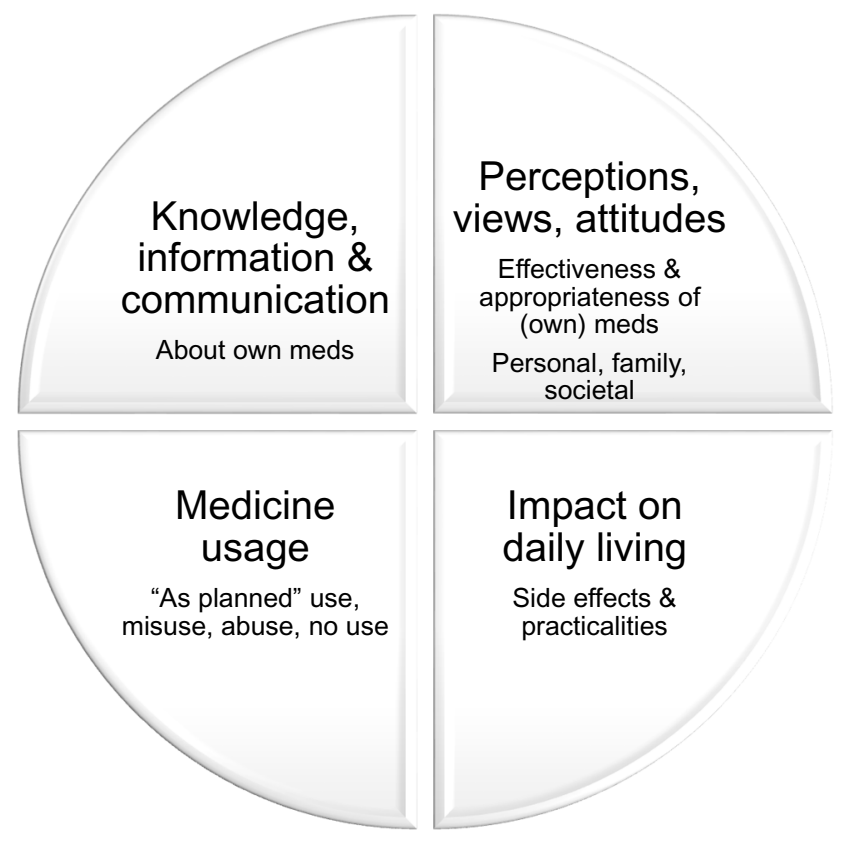

Figure 2 Thematic core categories of most meaningful/important PROs for assessment during polypharmacy medicines reviews.

Knowledge about one's own medicines, understanding the intended benefit of medicines, obtaining information and communicating about own medicines were considered the most important and meaningful PROs to be assessed during medicines reviews. Participants justified their views by raising points around the need to: Help people understand what medicines they take, what they are for, what they do, what the intended benefit is, and how to self-manage; Understand the need for more information and people's appetite for more information, while assessing any residual uncertainty; Have access trustworthy information about medicines; Ensure that the patient understands and is happy to follow the treatment plan; and Promote effective communication between patients/carers and healthcare professionals, and among providers and services.

Perceptions, Views and Attitudes About (Own) Medicines Perceptions and concerns about the appropriateness and effectiveness of prescribed medicines, including understanding people's personal goals and satisfaction with prescribed medicines were key PROs to be assessed in medicines reviews.

Participants talked about perceptions, views, goals and attitudes that, albeit important, are often undervalued or overlooked. A need for open conversations was indicated, where people can express whether they perceive benefit from their medicines, what they expect and want to achieve, how appropriate they deem their treatment plan, and what unhelpful personal, family or societal views and beliefs about medicines might interfere with the concordance of the treatment plan.

Impact on Daily Living: Side Effects and Practicalities Impact on daily living was considered from a dual viewpoint, the triggers being experiences of side effects and the practicalities of managing medicines (eg, fitting taking medicines into work/life schedule, availability and accessibility of medicines, or knowing who to report problems and how to reach them).

Participants felt strongly about the need to assess both of these sources of distress, any underlying factors (eg, lack of self-confidence or sense of control), as well as their implications for both patients and family members/carers, including ill health, impact on concordance of plan, impact on work and/or lifestyle.

Medicine Usage: “As Planned” Use, Misuse, Abuse, No Use

Members of the public and healthcare professionals equally expressed that monitoring and follow up (including issues around concordance, misuse, abuse and discontinuation) would be important to be included in PRO assessments during medicines reviews. The general view was that assessment of such PROs could help optimize the treatment plan as necessary, while considering the extent to which knowledge, perceptions or impact on daily living might play a role.

\section{Discussion}

In this study, we identified four core categories of most meaningful PROs to be assessed in polypharmacy medicines reviews (Figure 2), and 12 validated PROMs that, to different extents, can facilitate the assessment of the core PROs (Table 1). Diversity in our participants' demographic and clinical or professional characteristics catered for a rich description of experiences of using multiple medicines in Phase 2, which underpin evidence already reported in the literature. ${ }^{7,11,12}$ At the same time, our data directly linked to the 14 content domains extracted during the rapid review of PROMs in Phase 1. In line with previous evidence, ${ }^{34}$ members of the public described a multifaceted experience of managing and living with multiple medicines that was summarized in seven sub-themes. Healthcare professionals concurred with these views, except for the perceived 
embarrassment and stigma related to medicine use. These concepts have been previously investigated among people with mental health illnesses, ${ }^{35}$ and might be more prevalent among users of psychiatric medicines. $^{36}$

An overall need was expressed for easier navigation of the prescribing system, improved patient/family knowledge about the purposes and benefits of prescribed medicines, increased attention to the impact of medicines on daily living, and better collaboration and communication between patients/families and healthcare professionals, and across providers and services.

Effective use of PROMs was seen as conducive to addressing these needs. All participants agreed on the clinical relevance of PROMs, providing a rich account of justifications. In parallel, a good range of suggestions was made around usability and usage of PROMs to enhance integration and uptake in clinical practice. The consensus was that an effective buy-in process must involve:

- Consultation to identify best ways to introduce PROMs in clinical practice and best handlers of PRO information;

- A clear purpose for collecting PROMs;

- Realistic and ongoing collection at key time-points;

- PROMs that are easy to understand and not too long nor too wordy; and

- PROMs that only include key/essential and meaningful questions.

In relation to the latter point, participants identified a core set of four most meaningful PROs to be assessed in polypharmacy medicines reviews (Figure 2). Participants even went on to dwell about the potential interactions among these PROs, eg, how knowledge might interact with perceptions to affect usage, how impact on daily living can affect usage and vice versa, or how knowledge and communication can affect the degree of impact on daily living.

Participants collectively agreed that assessment of these four core PRO areas during medicines reviews can lead to focused and meaningful conversations, actions and interventions to tackle problematic polypharmacy (particularly in areas akin to prescription of psychiatric medicines and the associated health risks ${ }^{37,38}$ ), increase health literacy, optimize the treatment plan, and enable shared decision making and agreement to the prescribed (and optimized) treatment plan.

In terms of PROMs, in Phase 1, we searched the literature looking specifically for measures developed with direct user involvement. This was to increase our confidence in their clarity and relevance to future users in clinical practice. At the same time, we evaluated such aspects as length, comprehensiveness, and psychometric robustness of all measures. This was to shortlist measures, whose integration in clinical practice might be more feasible, as well as those that can return complete, valid and reliable information. To this end, we considered both single-domain and multi-domain measures. This was to examine the possibility for individual psychometrically robust domains to be used as stand-alone should a bespoke measure be required to be developed for clinical use out of individual domains of different existing PROMs.

At face value, PROMs varied widely in terms of length, comprehensiveness and psychometric robustness. Content validity through extensive literature reviews and direct and extensive user involvement at both item generation and clarification was established for the MedUseQ, ${ }^{30} \mathrm{PPDQ}^{33}$ and rPATD. ${ }^{28}$ In terms of likely feasibility for clinical use, the shortest measure was the MS-PETS ${ }^{32}(<10$ items) followed by the BMQ, ${ }^{29}$ PATD, ${ }^{27}$ PPDQ, ${ }^{33}$ TSQM v1, ${ }^{25}$ TSQM v2 $^{26}$ (all at 11-20 items). The most comprehensive measures (LMQ $3,{ }^{24}$ MedUseQ,${ }^{30}$ and $\mathrm{rPATD}^{28}$ ) tended to be lengthier, which could pose a barrier to their feasibility for clinical use. Even so, none of them covered all 14 of the content domains identified from the rapid review (see Table 1), or the four core thematic categories that were identified from the qualitative analysis of interview data.

Reliability and/or validity was fully established only for some PROMs, but no single PROM was fully validated across all of these parameters. Reliability (ie, internal consistency and stability) was fully established for the LMQ3 ${ }^{24}$ and PATD $^{28}$ only. Construct validity was fully established for the BMQ, ${ }^{29} \mathrm{LMQ}^{2},{ }^{24}$ and PETS ${ }^{32}$ only. Criterion validity was fully established for the MRB-QoL, ${ }^{31}$ followed by BMQ, ${ }^{29}$ TSQM v1, ${ }^{25}$ and TSQM v2. ${ }^{26}$ Across PROMs where an analysis for floor/ceiling effects was conducted, the MedUseQ ${ }^{30}$ was affected the least.

\section{Practice Implications}

All evidence considered, we recommend use of a bespoke PROM for clinical use, combining psychometrically robust existing PROMs or domains across existing PROMs (taking into consideration permission and copyright). To make it feasible for use in clinical practice, the bespoke PROM must be comprehensive enough to address all four core PRO areas, but not too long, ideally 
incorporating a maximum of $10-15$ items with $3-4$ items per core PRO area. ${ }^{39}$

Use of the bespoke PROM can follow a five-step process to ensure realistic integration in polypharmacy medicines reviews (Table 5). Moreover, to address concerns raised in this project about the disjointed healthcare system and lack of coherent communication flow across teams, disciplines, and settings we recommend further ongoing consultation to: (a) Identify appropriate timing and timepoints for collection of PRO information, eg, fixed timepoints at monthly intervals or every 6-12 months and/or on a "as and when" basis, eg, during initial prescription or when a change to the treatment plan is made. (b) Consider delivery mode of the bespoke PROM in different formats (paper or online), availability (in practice surgery, pharmacy or online, with one copy made available to the reporting patient or family), and healthcare professionals best placed to collect (ideally, everyone involved in the patient's treatment plan) and handle PRO information. (c) Exploreenhanced real-time and cross-discipline/setting

Table 5 Five-Step Process for the Use of a Bespoke PROM for Realistic Integration in Polypharmacy Medicines Reviews

\begin{tabular}{|c|c|}
\hline Steps & Description \\
\hline $\begin{array}{l}\text { Step I (clinical } \\
\text { relevance) }\end{array}$ & $\begin{array}{l}\text { Consider existing PROMs that have been } \\
\text { fully validated in terms of their content } \\
\text { validity (extensive literature review and } \\
\text { direct user involvement), including user } \\
\text { involvement at the generation and } \\
\text { clarification stage (ie, } \\
\text { MedUseQ, }{ }^{30} \text { rPATD }^{28} \text { and PPDQ } \\
\text { ). }\end{array}$ \\
\hline Step 2 (usability) & $\begin{array}{l}\text { Consider how appropriate the length of } \\
\text { the measures identified in Step I is. }\end{array}$ \\
\hline Step 3 (breadth) & $\begin{array}{l}\text { Consider the content domains that each of } \\
\text { the measures addresses, identifying } \\
\text { overlapping domains and domains that the } \\
\text { measures make a unique contribution to, } \\
\text { so that all four core PROs (and ideally all } \\
\text { I4 content domains) can be assessed by } \\
\text { the bespoke PROM. }\end{array}$ \\
\hline $\begin{array}{l}\text { Step } 4 \text { (degree of } \\
\text { established validity) }\end{array}$ & $\begin{array}{l}\text { Consider the extent of construct and } \\
\text { criterion validity undertaken for the } \\
\text { measures identified in Step I. Identify valid } \\
\text { stand-alone content domains of individual } \\
\text { PROMs. }\end{array}$ \\
\hline $\begin{array}{l}\text { Step } 5 \text { (content } \\
\text { validity) }\end{array}$ & $\begin{array}{l}\text { Develop draft bespoke PROM and initiate } \\
\text { consultation with stakeholders to establish } \\
\text { content validity in the first instance. }\end{array}$ \\
\hline
\end{tabular}

communication about prescribed medicines via an integrative online platform (or plug-in) that enables integration of PRO information into the patient's medical record and makes PRO information and prescription decisions/changes (perhaps via use of a flags system) available to all healthcare professionals involved in a patient's treatment.

\section{Strengths and Limitations}

In Phase 1, we followed a rigorous systematic approach to identify and select all studies that met our eligibility criteria, and synthesize evidence according to PRISMA guidelines ${ }^{17}$ and current guidance for rapid reviews. ${ }^{18}$ We conducted our synthesis of evidence in an unbiased manner to promote reproducibility. Some limitations of our sample of studies and review methodology must be acknowledged. We opted for an inclusive search strategy, but this was not exhaustive as it was limited to the most common databases. To abide by the timelines of a rapid review, we relied on the findings of a previous systematic review $^{8}$ for the period before January 2015. As such, our findings might be influenced by the methodological limitations of that systematic review; however, the extent of this is only minimal given the methodological rigor of that systematic review. We further limited our search to English language publications only. We cannot rule out the possibility that PROMs published in languages other than English might have been missed, but we anticipate that the number of these to be minimal.

In Phase 2, we opted for an inclusive recruitment strategy to increase diversity in our sample, which to a certain extent was achieved. Clear description of the context combined with a detailed account of participant characteristics enhances the transferability of our findings across NHS Scotland. Interviewers had no personal, clinical or professional relationship with the participants, which enhances the credibility of our data. However, specific limitations warrant comment. Due to time constraints, we opted for a convenience rather purposive sampling, which in conjunction to a small sample size, may limit the representativeness of experiences described in our sample. Specifically, our findings seem to be more skewed towards people already having positive views towards PROMs, patients rather than family members/carers, and GPs and pharmacists than registered nurses or medical consultants. However, our interviews did yield rather rich datasets with good variability of experiences, which was also confirmed by mapping findings to the 14 content domains of PROMs identified in Phase 1. 


\section{Conclusion}

All evidence considered, it is sensible to suggest combining psychometrically robust PROMs or domains across PROMs into a bespoke PROM that addresses the four core PROs of the multifaceted experience of polypharmacy in a comprehensive, yet succinct, way. A pragmatic approach to the clinical integration of a PROM (within NHS Scotland and beyond) will be essential to increase its uptake by healthcare teams and aid towards identification of medicine safety issues, prevention of inappropriate polypharmacy, and personalization of the treatment plan according to patients' and family members' and carers' changing needs and expectations.

\section{Ethics Approval}

Ethical approval was obtained from the University of Glasgow's School of Medicine, Veterinary and Life Sciences Ethics Committee (Ref. no.: 200180126; 30/04/19) to ensure that all ethical sensitivities were accounted for, and that appropriate standards were met in relation to key areas including confidentiality, anonymity and data protection.

\section{Acknowledgements}

We would like to thank Annabel Farnood (University of Glasgow) for her assistance with data collection during the qualitative interviews, and Paul Cannon (University of Glasgow) for his assistance with devising the search strategies for the rapid review.

\section{Author Contributions}

All authors made substantial contributions to conception and design, acquisition of data, or analysis and interpretation of data; took part in drafting the article or revising it critically for important intellectual content; gave final approval of the version to be published; and agree to be accountable for all aspects of the work.

\section{Funding}

Scottish Government Effective Prescribing and Therapeutics Division. The views expressed in the paper are those of the authors, not the funder.

\section{Disclosure}

The authors report no conflicts of interest in this work.

\section{References}

1. Johnston MC, Crilly M, Black C, Prescott GJ, Mercer SW. Defining and measuring multimorbidity: a systematic review of systematic reviews. Eur J Public Health. 2019;29(1):182-189. doi:10.1093/eurpub/cky098

2. Duerden M, Avery T, Payne R. Polypharmacy and medicines optimisation: making it safe and sound. Kings Fund. 2013 doi:10.1136/ bmjopen-2013-002913

3. Guthrie B, Makubate B, Hernandez-Santiago V, Dreischulte T. The rising tide of polypharmacy and drug-drug interactions: population database analysis 1995-2010. BMC Med. 2015;13(1):1-10. doi:10.1186/s12916-015-0322-7

4. Barrett K, Lucas E, Alexander GC. How polypharmacy has become a medical burden worldwide. Clin Pharm. 2016;8(6). doi:10.1211/ CP.2016.20201251

5. Cadogan CA, Ryan C, Hughes CM. Appropriate polypharmacy and medicine safety: when many is not too many. Drug Saf. 2016;39:109-116. doi:10.1007/s40264-015-0378-5

6. Eton D, Ridgeway J, Egginton J, et al. Finalizing a measurement framework for the burden of treatment in complex patients with chronic conditions. Patient Relat Outcome Meas. 2015;6:117-126. doi:10.2147/PROM.S78955

7. Sav A, King MA, Whitty JA, et al. Burden of treatment for chronic illness: a concept analysis and review of the literature. Heal Expect. 2015;18(3):312-324. doi:10.1111/hex.12046

8. Katusiime B, Corlett S, Reeve J, Krska J. Measuring medicine-related experiences from the patient perspective: a systematic review. Patient Relat Outcome Meas. 2016;7:157-171. doi:10.2147/PROM.S102198

9. Barnett K, Mercer SW, Norbury M, Watt G, Wyke S, Guthrie B. Epidemiology of multimorbidity and implications for health care, research, and medical education: a cross-sectional study. Lancet. 2012;380(9836):37-43. doi:10.1016/S0140-6736(12)60240-2

10. Payne RA. The epidemiology of polypharmacy. Clin Med J R Coll Physicians London. 2016;16(5):465-469. doi:10.7861/clinmedicine.16-5-465

11. Krska J, Morecroft CW, Poole H, Rowe PH. Issues potentially affecting quality of life arising from long-term medicines use: a qualitative study. Int $J$ Clin Pharm. 2013;35(6):1161-1169. doi:10.1007/s11096-013-9841-5

12. Moen J, Bohm A, Tillenius T, Antonov K, Nilsson JLG, Ring L. "I don't know how many of these [medicines] are necessary."-A focus group study among elderly users of multiple medicines. Patient Educ Couns. 2009;74(2):135-141. doi:10.1016/j.pec.2008.08.019

13. Eton D, Elraiyah TA, Yost K, et al. A systematic review of patient-reported measures of burden of treatment in three chronic diseases. Patient Relat Outcome Meas. 2013;4:7-20. doi:10.2147/ PROM.S44694

14. Beuscart JB, Knol W, Cullinan S, et al. International core outcome set for clinical trials of medication review in multi-morbid older patients with polypharmacy. BMC Med. 2018;16:21. doi:10.1186/s12916-018$1007-9$

15. Beuscart JB, Pont LG, Thevelin S, et al. A systematic review of the outcomes reported in trials of medication review in older patients: the need for a core outcome set. Br J Clin Pharmacol. 2017;83:942-952. doi:10.1111/bcp.13197

16. Scottish Government Polypharmacy Model of Care Group. Polypharmacy guidance, realistic prescribing. Scottish Government. 2018. Available from: https://www.therapeutics.scot.nhs.uk/wp-content/uploads/2018/09/ Polypharmacy-Guidance-2018.pdf. Accessed December 20, 2018.

17. Moher D, Liberati A, Tetzlaff J, Altman DG. Preferred reporting items for systematic reviews and meta-analyses: the PRISMA statement. BMJ. 2009;339(jul21_1):b2535. doi:10.1136/bmj.b2535 
18. Khangura S, Konnyu K, Cushman R, Grimshaw J, Moher D Evidence summaries: the evolution of a rapid review approach. Syst Rev. 2012;1:10. doi:10.1186/2046-4053-1-10

19. Mokkink L, Prinsen C, Patrick D, et al. COSMIN methodology for systematic reviews of Patient-Reported Outcome Measures (PROMs): user manual. Amsterdam Public Health Research Institute. 2018. Available from: https://www.cosmin.nl/wp-conten /uploads/COSMIN-syst-review-for-PROMs-manual_version-1_feb2018-1.pdf. Accessed April 30, 2019.

20. Maguire M, Delahunt B. Doing a thematic analysis: a practical, step-by-step guide for learning and teaching scholars. Irel J Teach Learn High Educ. 2017;8(3):3351-33514.

21. Carter SR, Bulanadi MG, Katusiime B, Chen TF, Corlett S, Krska J. Comprehensibly measuring patients' subjective thoughts, feelings and experiences of living with medicines: the Living with Medicines Questionnaire (LMQ). Int $J$ Clin Pharm. 2015;37:424-425. doi:10.1007/s11096-015-0080-9

22. Krska J, Morecroft CW, Rowe PH, Poole H. Measuring the impact of long-term medicines use from the patient perspective. Int $\mathrm{J}$ Clin Pharm. 2014;36(4):675-678. doi:10.1007/s11096-014-9970-5

23. Krska J, Katusiime B, Corlett SA. Validation of an instrument to measure patients' experiences of medicine use: the living with medicines questionnaire. Patient Prefer Adherence. 2017;11:671-679. doi:10.2147/PPA.S126647

24. Katusiime B, Corlett S, Krska J. Development and validation of a revised instrument to measure burden of long-term medicines use: the living with medicines questionnaire version 3. Patient Relat Outcome Meas. 2018;9:155-168. doi:10.2147/prom.s151143

25. Atkinson MJ, Sinha A, Hass SL, et al. Validation of a general measure of treatment satisfaction, the Treatment Satisfaction Questionnaire for Medication (TSQM), using a national panel study of chronic disease. Health Qual Life Outcomes. 2004;2(1):12. doi:10.1186/1477-7525-2-12

26. Atkinson MJ, Kumar R, Cappelleri JC, Mass SL. Hierarchical construct validity of the Treatment Satisfaction Questionnaire for Medication (TSQM version II) among outpatient pharmacy consumers. Value Heal. 2005;8(Suppl 1):S9-S24. doi:10.1111/ j.1524-4733.2005.00066.x

27. Reeve E, Shakib S, Hendrix I, Roberts MS, Wiese MD. Development and validation of the patients' attitudes towards deprescribing (PATD) questionnaire. Int $J$ Clin Pharm. 2013;35(1):51-56. doi:10.1007/s11096-012-9704-5

28. Reeve E, Low LF, Shakib S, Hilmer SN. Development and validation of the Revised Patients' Attitudes Towards Deprescribing (rPATD) questionnaire: versions for older adults and caregivers. Drugs Aging. 2016;33(12):913-928. doi:10.1007/s40266-016-0410-1
29. Horne R, Weinman J, Hankins M. The beliefs about medicines questionnaire: the development and evaluation of a new method for assessing the cognitive representation of medication. Psychol Heal. 1999;14(1):1-24. doi:10.1080/08870449908407311

30. Berman RL, Iris M, Conrad KJ, Robinson C. Validation of the MedUseQ: a self-administered screener for older adults to assess medication use problems. J Pharm Pract. 2018;8971900187. doi:10.1177/0897190018766789

31. Mohammed MA, Moles RJ, Hilmer SN, Kouladjian O’Donnel L, Chen TF. Development and validation of an instrument for measuring the burden of medicine on functioning and well-being: the Medication-Related Burden Quality of Life (MRB-QoL) tool. BMJ Open. 2018;8:e018880. doi:10.1136/bmjopen-2017-018880

32. Eton DT, Yost KJ, Lai JS, et al. Development and validation of the Patient Experience with Treatment and Self-management (PETS): a patient-reported measure of treatment burden. Qual Life Res. 2017;26 (2):489-503. doi:10.1007/s11136-016-1397-0

33. Linsky A, Simon SR, Stolzmann K, Meterko M. Patient perceptions of deprescribing: survey development and psychometric assessment. Med Care. 2017;55(3):306-313. doi:10.1097/MLR.0000000000 000642

34. Williams B, Shaw A, Durrant R, Crinson I, Pagliari C, de Lusignan S. Patient perspectives on multiple medications versus combined pills: a qualitative study. QJM. 2005;98(12):885-893. doi:10.1093/qjmed/hci139

35. Kamaradova D, Latalova K, Prasko J, et al. Connection between self-stigma, adherence to treatment, and discontinuation of medication. Patient Prefer Adherence. 2016;10:1289-1298. doi:10. 2147/PPA.S99136

36. Nelson E, Werremeyer A, Kelly GA, Skoy E. Self-stigma of antidepressant users through secondary analysis of PhotoVoice data. Ment Heal Clin. 2018;8(5):214-221. doi:10.9740/mhc.2018.09.214

37. Mojtabai R, Olfson M. National trends in psychotropic medication polypharmacy in office-based psychiatry. Arch Gen Psychiatry. 2010;67(1):26-36. doi:10.1001/archgenpsychiatry.2009.175

38. Gupta S, Cahill JD. A prescription for "deprescribing" in psychiatry. Psychiatr Serv. 2016;67(8):904-907. doi:10.1176/appi.ps.201500359

39. Johnston B, Flemming K, Narayanasamy MJ, Coole C, Hardy B. Patient reported outcome measures for measuring dignity in palliative and end of life care: a scoping review. BMC Health Serv Res. 2017;17:574. doi:10.1186/s12913-017-2450-6
Patient Preference and Adherence

\section{Publish your work in this journal}

Patient Preference and Adherence is an international, peer-reviewed, open access journal that focusing on the growing importance of patient preference and adherence throughout the therapeutic continuum. Patient satisfaction, acceptability, quality of life, compliance, persistence and their role in developing new therapeutic modalities and compounds to optimize clinical outcomes for existing disease states are major areas of interest for the journal. This journal has been accepted for indexing on PubMed Central. The manuscript management system is completely online and includes a very quick and fair peer-review system, which is all easy to use. Visit http:// www.dovepress.com/testimonials.php to read real quotes from published authors. 